\title{
SLEEP DISORDERS IN CHILDREN WITH EPILEPSY
}

Sleep and behavior disorders in 55 children with epilepsy (mean age 10 years; range 4-16 years) were compared with those in their non-epileptic siblings of the same ages and sex ratio and correlated with epilepsy-specific factors, in a study at Alberta Children's Hospital, University of Calgary, Alberta, Canada. Sleep Behavior Questionnaires (SBQ), Child Behavior Checklists (CBCL), and Quality of Life (QOL) Scores were completed by parents and caregivers. Epilepsy was idiopathic generalized in $8(15 \%)$, symptomatic generalized in $7(13 \%)$, and focal in $40(73 \%)$. Epileptiform discharges in the EEG were present in 51 patients, and in $62 \%$ of 39 with sleep records. Only 5 had nocturnal seizures. Seizures had been controlled for at least 3 months in $53 \%$, and for 1 year in $24 \%$. Treatment was monotherapy in $47 \%,>2$ AEDs in $38 \%$, and no AED in $15 \%$.

Children with epilepsy have significantly longer sleep latency, increased parent-child interaction at night, increased sleep fragmentation, increased parasomnias, and increased daytime drowsiness compared to non-epileptic siblings. They also score significantly higher on behavior subscales (withdrawn, somatic complaints, social problems, thought and attention problems, and aggressive behavior) compared to controls. Higher total sleep disorder scores are correlated with higher scores on CBCL subscales (withdrawn behavior, somatic complaints, social problems, and attention deficits), and significantly lower total QOL scores. Refractory epilepsy (35\%), mental retardation (53\%), and remote symptomatic etiology are risk factors for greater sleep problems with epilepsy. (Wirrell E, Blackman M, Barlow K, et al. Sleep disturbances in children with epilepsy compared with their nearestaged siblings. Dev Med Child Neurol Nov 2005;47:754-759). (Respond: Elaine Wirrell MD, Alberta Children's Hospital, 1820 Richmond Road SW, Calgary, Alberta, Canada T2T 5C7).

COMMENT. Children with epilepsy have increased sleep problems compared to nonepileptic siblings, and sleep disorders are correlated with daytime behavior problems and a poorer quality of life. Sleep patterns are an important question in the evaluation of children with epilepsy.

\section{HEADACHE DISORDERS}

\section{SLEEP DISORDERS IN CHILDREN WITH HEADACHE}

The prevalence of sleep disorders in 64 patients (38 [59\%] males and 26 [41\%] females; average age 10 years) with headache was evaluated in the Pediatric Neurology outpatient clinics, University of Chicago. Compared to 64 matched controls without headache, children with headache had a significantly higher prevalence of excessive daytime sleepiness, narcolepsy, and insomnia $(\mathrm{p}<0.005)$, but the prevalence of sleep apnea, parasomnias, and restlessness was not increased. In 18 patients diagnosed with migraine headaches, the scores for excessive daytime sleepiness and narcolepsy were significantly higher, but those for insomnia, parasomnia, sleep apnea, and restlessness were not different from non-headache controls. Medications did not alter the prevalence of any of the sleeping disorder symptoms $(\mathrm{p}>0.05)$. Headache patients had more frequent problems with 
concentration, urges to nap, sleeping at school, in the car, and a sensation of dreaming while awake $(\mathrm{p}<0.05)$. Headache patients with insomnia had trouble falling asleep, awakening suddenly after sleep onset, and feeling anxious about sleeping $(\mathrm{p}<0.05)$. Those with sleep apnea slept with mouths open $(\mathrm{p}<0.05)$. Headache patients had a more frequent history of seizures, dental problems, muscle disease $(\mathrm{p}<0.05)$, more frequent morning headaches, causing awakening, and nighttime arousals ( $\mathrm{p}<0.005$ ). (Luc ME, Gupta A, Birnberg JM, et al. Characterization of symptoms of sleep disorders in children with headache. Pediatr Neurol January 2006;34:7-12). (Respond: Dr Michel H Kohrman, Mc3055, University of Chicago, Dept of Pediatrics, 5841 S Maryland, Chicago, IL 60637).

COMMENT. A case history of a child presenting with headache should inquire about daytime sleepiness, narcolepsy, and insomnia. Children with headaches have more frequent symptoms of primary sleep disorders, and treating the sleep disorder may benefit the headache and improve the quality of life. Both headache and sleep disorders can be triggered by changes in neurotransmitters, and a fall in serotonin levels will affect REM sleep (Dodick DW et al. Headache 2003;43:282-292).

\section{TOPIRAMATE IN ALTERNATING HEMIPLEGIA OF CHILDHOOD}

A 12-year-old girl with a family history of migraine and attacks of hemiplegia was treated successfully with topiramate (TPM), in a report from the University Hospital of Messina, Italy; and Leiden University Medical Centre, The Netherlands. Hemiplegic attacks began at 7 months of age, they averaged 2 or 3 times per week, lasted 24 to 36 hours, resolved with sleep, shifted from one side to the other, and were associated with pendular nystagmus. Attacks were triggered by emotional stimuli or fever. Her psychomotor development was delayed; she talked at 3 years and walked at age 4.5 years. A video-EEG showed posterior slow waves during an attack of hemiplegia. At follow-up she had dystonia, dysarthria, ataxia, and pyramidal tract signs. Treatment with valproate, carbamazepine, and benzodiazepine failed to control attacks, and flunarazine $5 \mathrm{mg} /$ day reduced duration of attacks to 2 to 3 hours for 5 months, Attacks and motor and social skills worsened, and her IQ measured by the WISC was 48 . TPM was introduced, $1-3 \mathrm{mg} / \mathrm{kg} /$ day, with a prompt reduction in severity and frequency of attacks, and continued improvement of dystonia and ataxia through 18-month follow-up. The mechanism of action was attributed to the inhibition of carbonic anhydrase. (Di Rosa G, Spano M, Pustorino G, et al. Alternating hemiplegia of childhood successfully treated with topiramate: 18 months of follow-up. Neurology January (1 of 2);66:146). (Reprints: Dr Gabriella Di Rosa, Medical and Surgical Pediatrics, Division of Infantile Neuropsychiatry, University Hospital of Messina, via Consolare Valeria 98125, Messina, Italy).

COMMENT. Alternating hemiplegia of childhood (AHC) typically begins in infancy, and the etiology is unknown. It resembles migraine and familial hemiplegic migraine (FHM), but only a few familial cases of $\mathrm{AHC}$ have been reported (Mikati MA et al. Neurology 1992;42:2251-2257; Andermann E et al. Neurology 1994;44:1812-1814; Sakuragawa N. Brain Dev 1992;14:283-288; Ped Neur Briefs Jan 1993 and Nov 1994). Flunarazine is the most frequently used treatment, with variable response. In the present case, TPM was effective and deserves further study. 